\section{Schlechte Bakterien stören die Wundheilung}

Durch eine Darmoperation kann sich die Zusammensetzung der Darmbakterien so verändern, dass sie giftige Substanzen freisetzen und die Wundheilung stören. Das konnten Wissenschaftler um John Alverdy, Universität Chicago, im Tiermodell zeigen. Das Ziel müsse daher sein, die "guten" Bakterien im Darm zu fördern und die "schlechten" zu verdrängen. Die Wissenschaftler forschen nun an einem Wirkstoff, der anders als Antibiotika keine Resistenzen fördert. Sie hoffen, so den Antibiotika-Einsatz zu reduzieren und multiresistente Krankenhauskeime in den Griff zu bekommen.

\section{Sci Transl Med. 2015;7:286ra68}

\section{Schlechte Prognose}

Junge Schlaganfallpatienten bleiben langfristig auf Hilfe im Alltag angewiesen. Die Aussichten für Frauen sind besonders schlecht. 14 Jahre nach dem Ereignis war in einer niederländischen Untersuchung jeder fünfte Überlebende von fremder Hilfe abhängig. Das berichten Ärzte um Nathalie Synhaeve von der Universitätsklinik in Nijmegen. Als Risikofaktoren für eine ungünstige Langzeitprognose erwiesen sich weibliches Geschlecht und der Schweregrad des Schlaganfalls.

J Neurol 2016, online 2. April

\section{Verdünnter Apfelsaft statt Elektrolytlösung}

Die orale Rehydratation führt bei Kindern mit leichter Gastroenteritis eher zum Erfolg, wenn statt spezieller Elektrolytlösungen verdünnter Apfelsaft gegeben wird, haben kanadische Ärzte in einer randomisierten Studie mit 647 Kindern gezeigt. Die Kinder zwischen 6 und 60 Monaten waren mit Gastroenteritis und leichter Dehydratation in eine Notfallambulanz gebracht worden. 323 wurden mit einem 1:1-Mix aus Apfelsaft und Wasser rehydriert, 324 bekamen eine gleichfarbige Elektrolytlösung mit Apfelgeschmack. Mit der Apfelsaft-Strategie kam es bei deutlich weniger Kindern innerhalb von sieben Tagen zu einem Therapieversagen ( $16,7 \mathrm{vs}$. 25,0\%). Als gescheitert galt die Behandlung u.a. dann, wenn die Patienten i.v. rehydriert oder stationär aufgenommen werden mussten, oder wenn ein Gewichtsverlust von mindestens 3\% festgestellt wurde.

JAMA 2016;315(18):1966-1974

\title{
Der Dauerkatheter als Problemschlauch
}

Den Anteil der Patienten, die einer langfristigen Harnableitung mit einem
Blasenkatheter bedürfen, schätzt Prof. Florian Wagenlehner, Gießen, auf $15-25 \%$ der langzeitig stationär Behandelten und auf 5-10\% der in Pflegeheimen Betreuten. Den behandelnden Ärzten sei oft gar nicht bekannt, dass ihre Patienten einen Dauerkatheter haben. Auch seien die Indikationen häufig nicht angemessen, vor allem in Krankenhäusern.

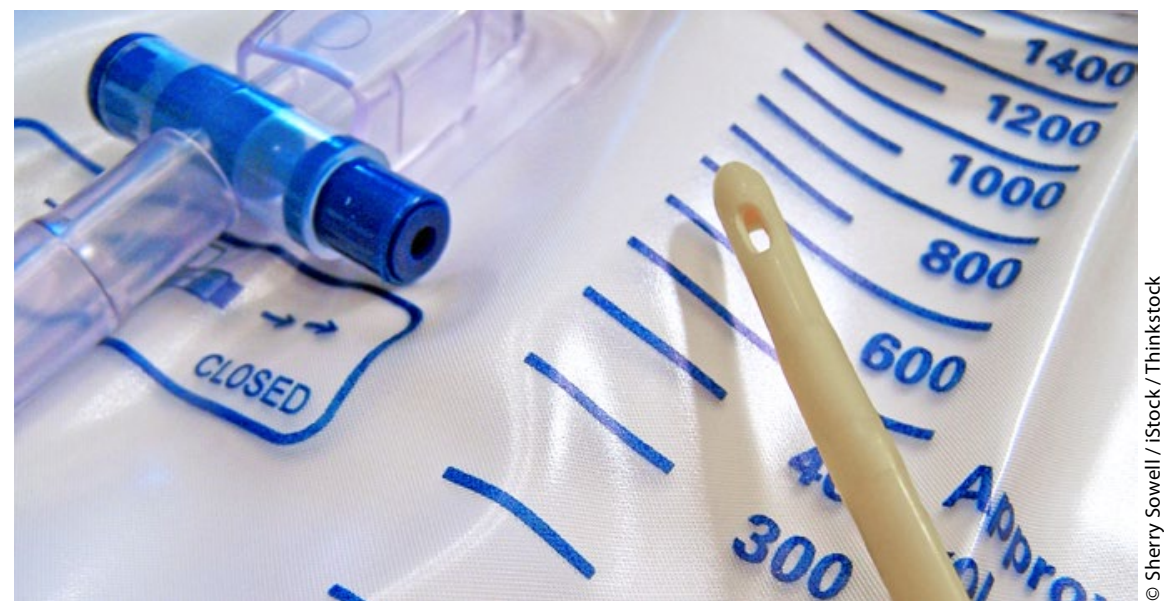

D ie Nachteile der katheterunterstützten Harnableitung sind bekannt: blockierte Harnableitung, Fisteln, Stenosen, Strikturen und natürlich katheterassoziierte Harnwegsinfektionen bis hin zur Urosepsis können die Folge sein. Über lange Zeit - das heißt hier: fünf bis zehn Jahre - liegende Katheter erhöhen auch das Blasenkrebsrisiko. Praktisch alle katheterisierten Patienten entwickeln auf die Dauer eine symptomatische bakterielle Infektion. Bereits zwei bis drei Tage nach Einlage eines Katheters entsteht eine signifikante Bakteriurie. Die Infektionswege verlaufen intraund extraluminal. In 35\% der Fälle handelt es sich bei den besiedelnden Keimen um endogene, in $40 \%$ um exogene Keime, bei den übrigen ist die Herkunft unbekannt. Als Risiken gelten höheres Alter, längere Liegedauer des Katheters, weibliches Geschlecht und geschwächte Immunabwehr. Probleme bereitet besonders die Bildung von Biofilmen. In solchen sind die Keime der körpereigenen Abwehr entzogen, und auch antimikrobiell wirkende Substanzen können ihnen wenig anhaben.

Viel ist deshalb versucht worden, der Infektionsgefahr durch spezifische Beschichtung der Blasenkatheter Herr zu werden - doch vergeblich. Ob Silberlegierung oder Nitrofural - es gab keine statistisch signifikanten Unterschiede mit Blick auf das Auftreten symptomatischer Harnwegsinfektionen. Insgesamt könne den beschichteten Kathetern in dieser Hinsicht kein präventiver Nutzen zugeschrieben werden, so Wagenlehner. Er empfiehlt, präventiv zunächst einmal die Indikation zur Katheterisierung sorgfältig zu stellen. Kontinenzmanagement beispielsweise sei keine Indikation für das Legen eines Dauerkatheters. Behandelnde Ärzte müssten die Katheter anordnen und wissen, welche Patienten und wie lange diese schon einen Katheter hätten. Nützlich seien bildgebende Untersuchungen der Harnblase, um die Restharnmengen zu bestimmen. Blasenkatheter sollten so früh wie möglich gezogen werden; dabei seien automatisierte Erinnerungssysteme hilfreich. Gemäß den von Wagenlehner vorgelegten Daten, wären Harnwegsinfektionen mit solchen Kathetern nicht seltener als mit über die Harnröhre geschobenen Ableitungen.

(rb)

"State-of-the-art lecture: Long-term catheterization and its problems", F. Wagenlehner, EAUKongress; München, 14. März 2016 